\title{
Calculation and Simulation of Transmission Reliability in Wireless Sensor Network Based on Network Coding
}

\author{
https://doi.org/10.3991/ijoe.v13i12.7883 \\ Zhifu Luan \\ Weifang University of Science and Technology, Weifang, China \\ 912268476 @qq.com
}

\begin{abstract}
The wireless sensor network (WSN) has penetrated into every corner in our lives, ranging from national defense, biological medicine, environmental monitoring, to traffic management. It is of great significance to study the reliability of data transmission, a key determinant of the results of monitoring events. The network reliability lies in fault tolerance: when some nodes or links in the network fail, the data can be recovered at the sink node by selecting the appropriate finite domain space. In this paper, network coding is used to improve the reliability of WSN. Firstly, the author calculated the data transmission reliability and average energy consumption of network coding in singlepath and multi-path scenarios. Then, the average energy consumption of network coding was compared with that of the traditional method. Finally, the reliabilities of the two different methods were simulated on MATLAB at different channel loss rates. The experimental results show that the reliability of the network coding technique is better than the traditional one at the expense of a small amount of energy.
\end{abstract}

Keywords-Wireless Sensor Network (WSN), Network Coding, Reliability

\section{$1 \quad$ Introduction}

The wireless sensor network (WSN) has penetrated into every corner in our lives, ranging from national defence, biological medicine, environmental monitoring, to traffic management. The network is formed through self-organization of sensors deployed in multiple sensing areas. In the WSN, the sensors can work cooperatively to sense, collect and process specific information in the monitoring area, laying the basis for real-time acquisition, processing and transmission of information [1]. It is of great significance to study the reliability of data transmission, a key determinant of the results of monitoring events.

The reliability of data transmission is an integral part of network reliability. The research on network reliability can be traced back to the 1960s. However, the early research mainly focused on communication networks, power networks and computer networks. Originated from network reliability, the WSN reliability has not been uniformly defined yet. In Reference [2], the concept is defined as the probability of normal communication between nodes in the network under the given constraints. Refer- 
ence [3] gives the definition of the ability to deliver material, information and energy under the specified conditions and within a desired time frame. Reference [4] defines WSN reliability as the success rate of source-to-destination data transmission in the network. Over the years, various models have been constructed to study the WSN reliability. For example, Reference [5] constructs a Markov chain-based reliability model in light of the state transition in both software and hardware. The model is further improved in Reference [6] with a quantitative analysis method. References [7, 8] introduce a reliability modelling method based on Architecture Analysis \& Design Language (AADL), which quantifies the reliability following the idea of the Generalized Stochastic Petri Nets (GSPN) model.

The WSN reliability is mainly reflected by such three parameters as data acquisition, data transmission and network coverage [9]. Among them, the data transmission plays the most fundamental role $[10,11]$. Traditionally, the reliability of data transmission in the WSN are improved by Automatic Repeat Request (ARQ), Forward Error Correction (FEC) and multi-path transmission. The reliability of data acquisition is often modelled as an overlay problem [12], while the network coverage is a reflector of the monitoring quality and the reliability of the acquired data. By definition, network coverage refers to the ability to monitor each location in an area of interest. The greater the network coverage, the greater the monitoring ability. The parameter is often measured by the K-coverage [13, 14], that is, the location or area of interest is covered by at least $\mathrm{K}$ nodes. To meet different data reliability requirements in the area of interest, the K value can be set according to the unused Quality of Service (QoS).

Data acquisition is one of the many functions of the WSN. The successful operation of the WSN also relies on the reliable transmission of the acquired data to the observer. However, the reliability of data transmission is traditionally explored based on the reliable routing mechanism, and rarely from the perspective of network topology. The one-sided focus on reliable routing mechanism is attributable to the fixed topology of traditional networks. As sensor nodes grow denser in distribution and greater in quantity, the network topology has become increasingly flexible, making it possible to study data transmission reliability based on network topology [15-17].

In light of the above, this paper first introduces the composition and characteristics of the WSN, and then calculates the reliability and average energy consumption of the network under single-path and multi-path conditions. Next, the network coding technique was compared with a traditional method in terms of the average energy consumption. Finally, the reliabilities of the two different transmission plans at different channel loss rates were obtained by MATLAB simulation. The experimental results show that the network coding technique achieves better stability than the traditional mode at the expense of a small amount of energy, and that network coding is superior to the traditional mode in multi-path routing. 


\section{Composition and Characteristics of the WSN}

\subsection{Composition of the WSN}

The WSN is a task-oriented wireless ad hoc network [18], that integrates sensor technology, embedded technology, wireless communication technology with distributed information processing technology. The network monitors the target in real time with various micro sensors, processes the monitoring data in an embedded module, and transmits the data to the remote monitoring centre via wireless or physical network. The WSN consists of four parts: wireless sensor nodes, sink node, transmission network, and remote monitoring centre. Its structure is shown in Figure 1.

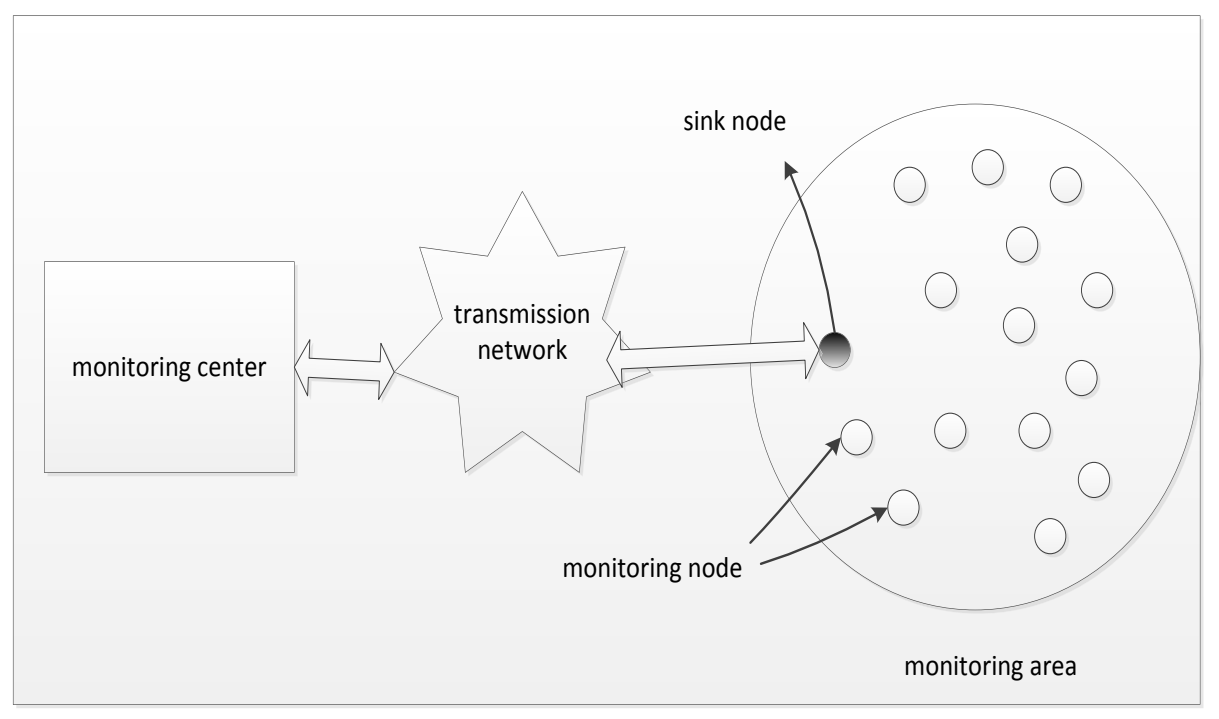

Fig. 1. The structure of WSN

\subsection{Characteristics of the WSN}

The WSN, as the combination of various emerging technologies, carries many characteristics that are not available in general networks [19].

1. Self-organization: the WSN is an ad hoc network formed with sensor nodes based on the distributed network protocol. Without relying on any fixed infrastructure, the network automatically adjusts the entry, exit and migration of nodes. In this way, a plurality of active nodes can quickly form an independent network.

2. Decentralization: all nodes in the WSN have equal status and form a peer-to-peer network. The nodes can join or leave the network momentarily at any time if necessary. The status of some nodes does not affect the operation of the entire network. 
3. Dynamic topology: In the case of battery exhaustion or failure, the WSN nodes may exit the network following the pre-set procedures. Owing to the mobility of sensor nodes, external wireless sensor nodes can be admitted to the network at any time.

4. Multi-hop routing: The internodal communication distance is relatively short in the WSN. The nodes can only communicate directly with neighbouring nodes. Distant communication requires an intermediate node for data transmission. The multi-hop routing in the WSN is accomplished by common nodes, each of which both receives and forwards information. The data is transmitted from each sensor node to the sink node via multi-hop relay.

5. Redundant height: For the sake of system reliability and fault tolerance, the same monitoring point is covered by the monitoring ranges of multiple sensor nodes.

6. Limited hardware resources: Under the constraints of price, volume and power consumption, the nodes have much smaller computing power, program space and memory space than ordinary computers. This excludes complex protocol hierarchy from the design of node operating system.

7. Limited power supply: The sensor nodes are powered by battery, which cannot be charged or replaced in many applications. When the battery runs out, the nodes will lose their functions. Thus, energy-saving must be considered in the design of the WSN.

8. Fault tolerance: The WSN boasts strong fault tolerance and robustness in that the nodes can join or exit the network at any time, and that the network operation is immune from the fault of any node.

9. Broadcast communication: the broadcast communication mode is adopted instead of the point-to-point mode in traditional network.

10. Data processing: The traditional emphasis on connectivity is replaced by the focus on data. The WSN nodes are required to possess such functions as data aggregation, fusion, capturing and compression.

\subsection{Energy consumption of sensor nodes}

The highlighting of energy-saving differentiates the WSN from traditional wireless networks. The energy saving is no big deal in traditional wireless networks due to continuous energy supplementation. By contrast, the WSN faces serious energy constraint because of the large number of widely distributed sensor nodes. Therefore, it is the primary goal of WSN design to make efficient use of node energy and prolong the life of the network.

There are three parts in the energy consumption module of sensor nodes, namely sensor module, processor module and wireless communication module. All these modules should be designed for the purpose of energy-saving. Hereinto, the power consumption of the processor module depends on four factors: operating voltage, operating clock, internal logic complexity, and manufacturing process, and calls for the development of energy-efficient microprocessors and microcontrollers. Thanks to the recent improvements in low-power circuits and system design techniques, it is now possible to develop ultra-low-energy microprocessors and micro-controllers. The 
progress of integrated circuit has further lowered the energy consumption of processors and sensor nodes, making the wireless communication module the largest consumer (Figure 2).

The wireless communication module has four states, including sending, receiving, idle and sleeping. In the idle state, the module always monitors the use of wireless channel and checks if data have been sent to itself. In the sleeping state, the communication module is shut down. The sending state marks the greatest energy consumption of the module, followed closely by the idle and receiving states. By contrast, the module has the minimal energy consumption in the sleeping state. Hence, the network communication can be made more energy-efficient by reducing unnecessary forwarding and receiving, and speeding up the activation of the sleeping state. The efficient use of energy may help to maximize the lifetime of the WSN.

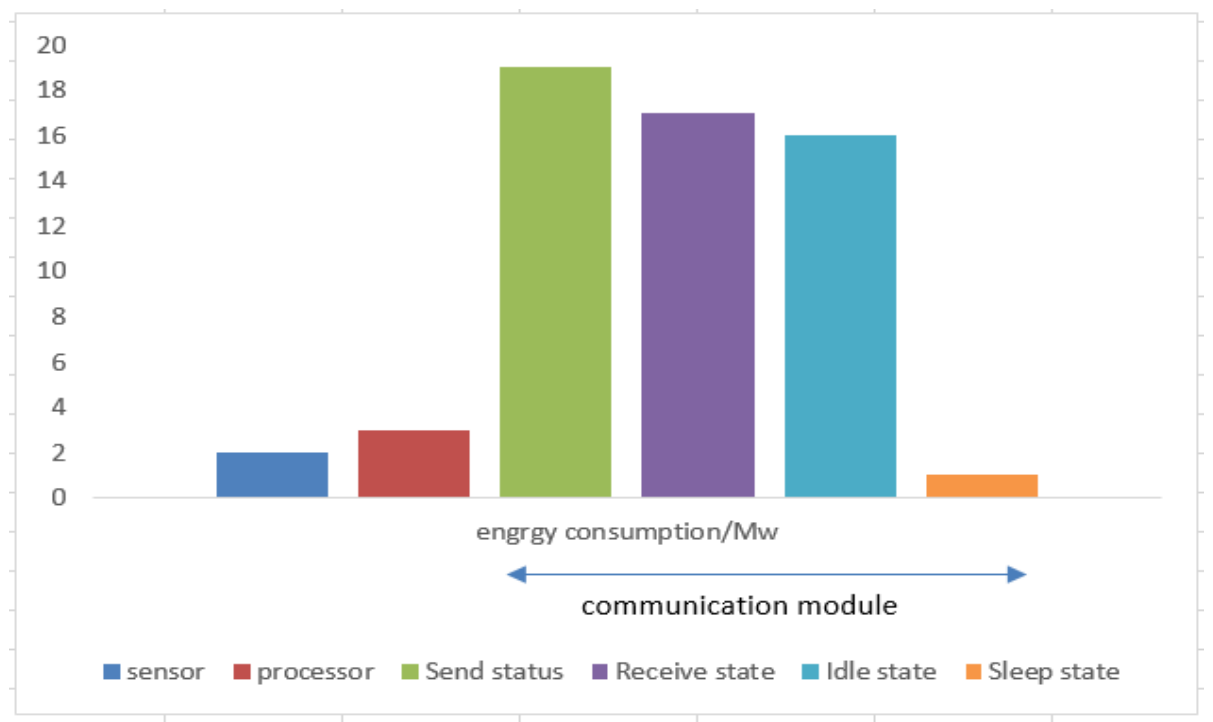

Fig. 2. The energy consumption module of sensor nodes

\section{Transmission Reliability of Network Coding}

In the WSN, reliable data transmission can be achieved at elevated redundancy. For example, the ARQ transfers data packets by retransmission, the FEC adds redundant packets to the source node, and the multi-path method transmits redundant packets via multiple paths. Moreover, the reliability of WSN data transmission is easily affected by the quality of internodal communication. One of the most common ways to enhance data transmission reliability lies in the reduction of channel loss rate with redundant packets. The simplest topology of the WSN is the star network, and the reliability model of the same network is different under different requirements (Figure 3). 


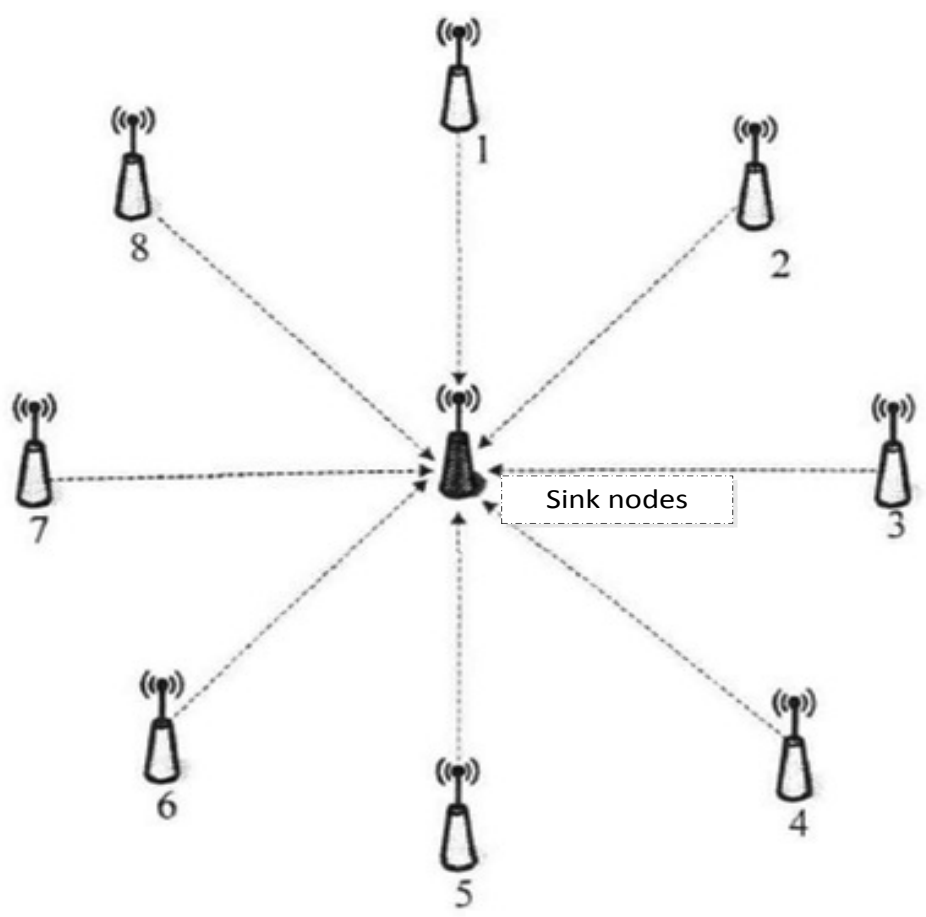

Fig. 3. Star network

If network coding technique is used alone, it is impossible to avoid the channel loss effect. After all, the effect is the same whether the sink node loses one packet only or all packets at once. Thus, the network coding technique should be

combined with the redundant technology to improve data transmission reliability. The popular ways to increase redundancy include multi-path transmission, retransmission coding of data packets, and increasing redundant packets.

The FEC is encoded only at the source node and decoded at the destination node. In network coding, however, the intermediate nodes can be recoded. Compared with FEC, the network coding features better flexibility and fault tolerance. For example, assume that 3 raw data packets are transmitted such that the last-hop node of the sink node receives the encoded data packets $\mathrm{x} 1, \mathrm{x} 2, \mathrm{x} 3$ and $\mathrm{x} 4$, where $\mathrm{x} 1$ is linearly correlated with the encoding vector of $\mathrm{x} 2$, and other data packets are linearly independent. If the FEC is adopted, the node will forward the packets directly. Suppose the packet $\mathrm{x} 3$ is lost after transmission, the sink node will not be able to decode the data packets $\mathrm{x} 1, \mathrm{x} 2$ and $\mathrm{x} 4$ because the encoding coefficient matrix is not full rank. If the network encoding is employed, the node will receive the 4 new encodings by encoding the data packets, and the coefficient matrix of any three of these packets is full rank. Even if a packet is lost, the sink node can decode it smoothly.

To disclose the advantage of network coding, the reliability of data transmission and energy consumption of network coding were simulated with different paths. The 
reliability of data transmission is denoted as $\mathrm{R}$ and defined as the probability that the sink node will receive the original data packet successfully after one transmission.

Definition 1: The average energy consumption E refers to the average energy consumed by a successfully transmitted packet. It can be expressed as:

$$
E_{\text {average }}=\frac{\text { Total energy consumed }}{\text { Number of packets received }}
$$

Definition 2: The ratio of the average energy consumption in network coding to that in the traditional mode can be expressed as:

$$
\eta=\frac{E_{\text {average }}^{\text {c }}}{E_{\text {average }}^{t}}
$$

\subsection{Single-path transmission}

In a multi-hop WSN, the packets are transmitted by sensor nodes via multi-hop routing, the internodal communication quality is susceptible to channel effects, and link failures are independent of each other. In the case of link failure, the sensor nodes will not fail. Let us denote the channel loss rate as e, and the energy consumed by a node to send a packet ase. Through an $n$ time hopping, the probability for a packet to reach $\mathrm{p}$ nodes is:

$$
p=(1-e)^{n}
$$

Assuming that the source node sends $K$ packets, the probability for the sink node to successfully receive $K$ packets is:

$$
P=(1-e)^{n K}
$$

where the probability $\mathrm{P}$ is equivalent to the reliability $\mathrm{R}$ defined in the preceding section.

The energy consumed to send a packet to the sink node via an $\mathrm{n}$ time hopping is calculated as follows. Since the packet is lost after a certain hop, the total energy consumed to send $K$ packets can be obtained by induction.

$$
E_{t}=K n \varepsilon(1-e)^{n}+\sum_{i=1}^{n} \varepsilon i K(1-e)^{i-1} e
$$

The number of packets received by the sink node is expected to be Kp, whereby the average energy consumption is:

$$
E_{\text {average }}^{t}=\frac{E_{t}}{K p}=\frac{K n \varepsilon(1-e)^{n}+\sum_{i=1}^{n} \varepsilon i K(1-e)^{i-1} e}{K(1-e)^{n}}=n \varepsilon+\sum_{i=1}^{n} \varepsilon i(1-e)^{i-1-n} e
$$


The average energy consumption ratio is:

$$
\eta=\frac{E_{\text {average }}^{c}}{E_{\text {average }}^{t}}=\frac{(n+1) \mu}{\left[n \varepsilon+\sum_{i=1}^{n} \varepsilon i(1-e)^{i-1-n} e\right](K+m)(1-e)^{n}}+1
$$

By Formula (7), it is learned that $\eta>1$. In this research, random linear coding is used for the simple computation of nodes.

\subsection{Multi-path transmission}

Suppose there exist L non-intersecting paths which are of equal length n, and the source node sends K packets simultaneously through multiple paths to the sink node. Then, the probability for any packet to successfully reach the sink node after one transmission is:

$$
p=1-\left[1-(1-e)^{n}\right]^{L}
$$

Thus, the probability for the sink node to successfully receive $\mathrm{K}$ packets is $\mathrm{Kp}$, that is, the reliability $\mathrm{R}$ is,

$$
P_{k}=p^{K}=\left\{1-\left[1-(1-e)^{n}\right]^{L}\right\}^{K}
$$

If $K$ packets are sent along the $\mathrm{L}$ paths, the total energy consumption is:

$$
E_{t}^{\prime}=L E_{t}
$$

The average energy consumed to send the $K$ packets along the $L$ paths is:

$$
E_{\text {average }}^{t}=\frac{E_{t}{ }^{\prime}}{L K(1-e)^{n}}=\frac{E_{t}}{L K(1-e) n}=E_{\text {average }}^{t}
$$

When the network coding is employed in multi-path transmission, the intermediate nodes only encode without increasing redundant packets. In this case, the total energy consumed to send $K$ packets is:

$$
E_{c}{ }^{\prime}=L\left[K n \varepsilon(1-e)^{n}+\sum_{i=1}^{n} \varepsilon i K(1-e)^{i-1} e\right]+[(n-1) L+2]
$$

The number of packets received by the sink node is expected to be:

$$
L K(1-e)^{n}
$$

Therefore, the average energy consumption is:

$$
E_{\text {average }}^{c}=\frac{E_{c}{ }^{\prime}}{L K(1-e)^{n}}
$$


The average energy consumption ratio $\eta_{\text {is: }}$

$$
\eta^{\prime}=\frac{E_{\text {average }}^{c}{ }^{\prime}}{E_{\text {average }}^{{ }^{\prime}}}=\frac{E_{c}{ }^{\prime}}{E_{t}{ }^{\prime}}=1+\frac{[(n-1) L+2] e}{3000 L K\left[1+n(1-e)^{n+1}+(n e-n-1)(1-e)^{n}\right]}
$$

\section{Simulation Experiment and Result Analysis}

The model given in the previous section was simulated in the environment of MATLAB 7.0.

\subsection{Single-path transmission}

Suppose that the path length is 5 , and the source node sends 3 packets to the sink node such that the sink node receives 5 encoded data packets. Then, the reliabilities of the network coding and the traditional method were simulated at different channel loss rates.

As shown in Figure 4, the network coding has achieved better reliability than the traditional method. This paper only considers the redundant coded packets at the source node. The reliabilities of the two methods still decreased with the loss of channel. In a single-path, the network coding improved the reliability to a greater extent than the traditional method at high channel loss rate of redudant data and with few network encoding packets; however, the results were still not desirable. To fruther improve the data transmission reliability of network coding, appropriate redundant data packets should be added to the nodes.

Figure 5 presents the average energy consumption ratio of the two different transmission methods. It can be seen that $\eta$ increased with e. However, regardless of the size of the e, the energy consumed by the single-path transmission combined with network coding always exceeded the energy consumed by that combined with the traditional method. This means network coding can improve the reliability of data transmission in the network at the expense of a small amount of energy.

\subsection{Multi-path transmission}

Similarly, assume that 5 packets are sent from the source node along 3 paths, each of which is 5 in length. Then, the reliabilities of the network coding and the traditional method were simulated at different channel loss rates.

As can be seen from Figure 6, the network coding method outperformed the traditional method at the same redundancy. With the increase of the channel loss rate, both method transmitted fewer redundant data, indicating that the transmission improvement failed to offset link failure and results in longer paths.

Figure 7 illustrates the average energy consumption ratio between the two different methods. It shows that network coding can improve the reliability of data transmission in the WSN at the expense of a small amount of energy. 


\section{Conclusions}

This paper calculates and analyses the transmission reliability and average energy consumption of network coding in single-path and multi-path scenarios. The simulation on MATLAB 7.0 indicates that the technique is more reliable than the traditional method at the expense of a small amount of energy. In addition, the author also simulated the reliabilities and the average energy consumption ratio of the network coding and the traditional method. The experimental results verify that the network coding technique is superior to traditional transmission.

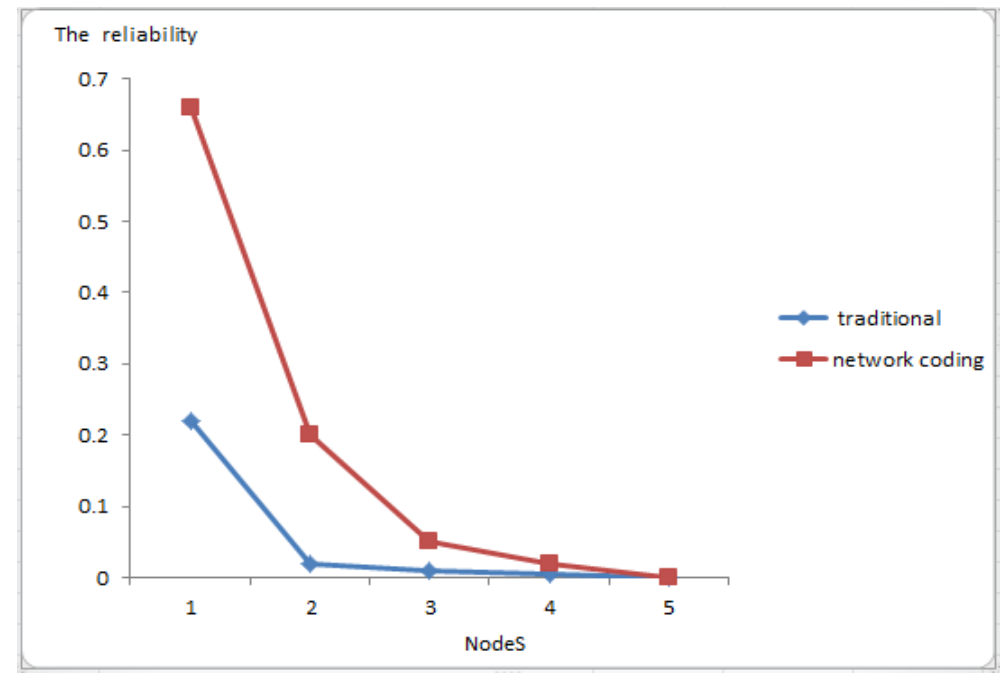

Fig. 4. The reliabilities of the two methods

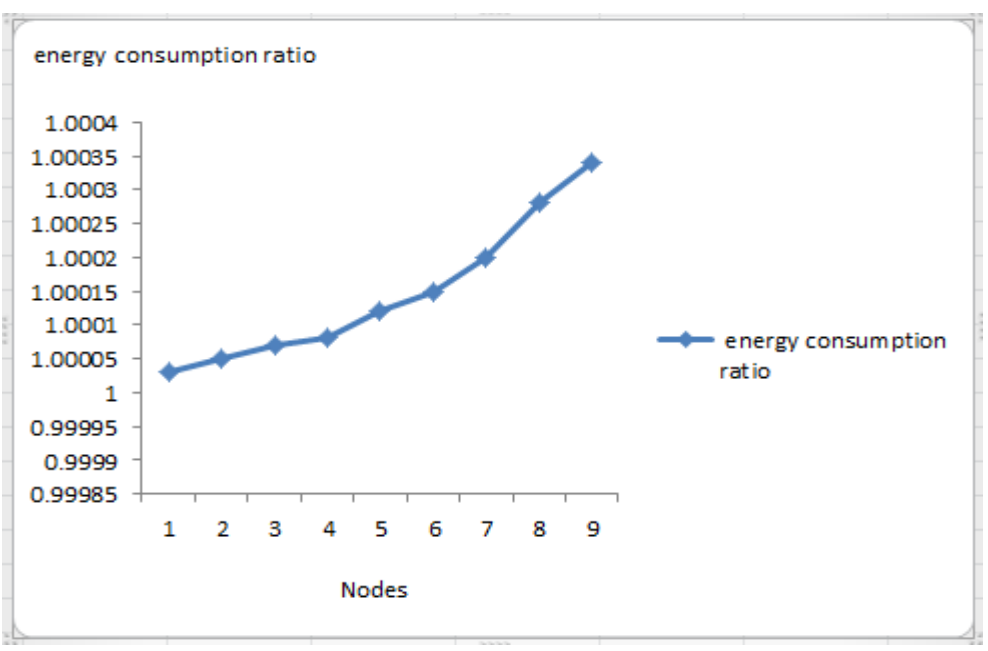

Fig. 5. The average energy consumption ratio of the two methods 


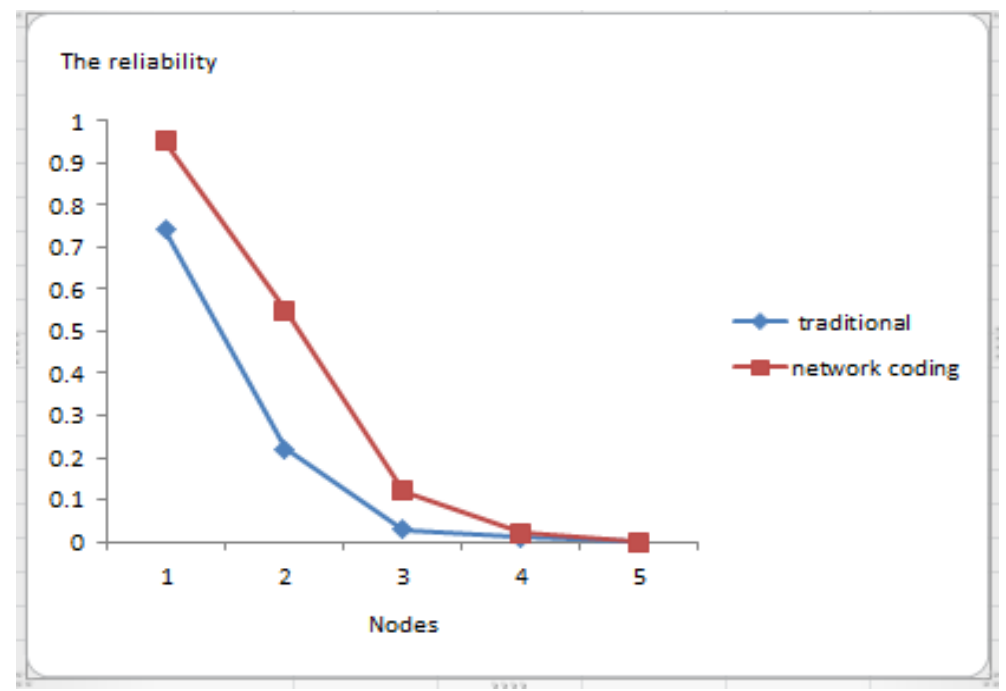

Fig. 6. The reliabilities of the two methods

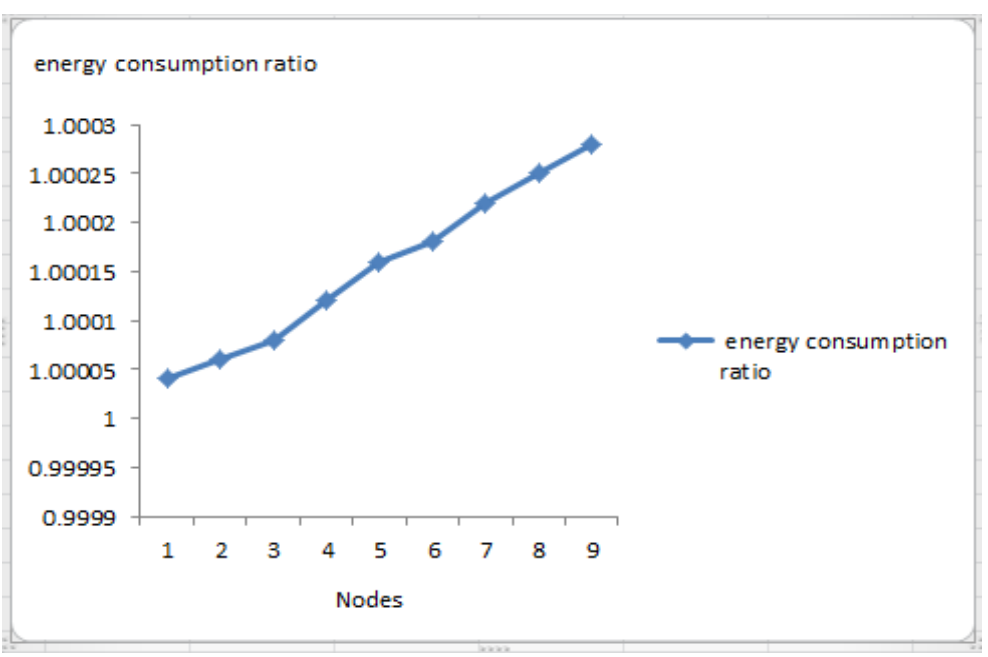

Fig. 7. The average energy consumption ratio of the two methods

\section{References}

[1] Sun L.M., Li J.Z. (2005). Wireless Sensor Networks, Beijing: Tsinghua Press.

[2] Meng H.J. (2010). Research on Optimization of network reliability and its application, Shanghai: East China University of Science and Technology.

[3] Li R.Y., Huang N., Chen W.W. (2009). A Practical Approach for Network Application Reliability Assessment, Ek-sploatacja Iniezawodnosc-Maintenance and Reliability, 4, pp. $17-27$. 
Paper-Calculation and Simulation of Transmission Reliability in Wireless Sensor Network Based on...

[4] Yousefi H., Mizanian K., Jahangir A.H. (2010). Modeling and Evaluating the Reliability of Cluster-Based Wireless Sensor Networks, Proceedings of 24th IEEE International Conference on Advanced Information Networking and Applications. https://doi.org/10.1109/ AINA.2010.72

[5] Tang F., Luo H.W., Xu Y.W. (2005). The application of Mobile Ad Hoc network in the US, Engineer equipment research, 24(5), pp. 61-64.

[6] Rolader G., Rogers J.C., Batteh J. (2004). Self-healing minefield, Pro-ceedings of the Society of Photo-Optical Instrumentation Engineers. Bellingham, USA, pp. 13-24.

[7] Huang C.F., Tseng Y.C. (2005). A survey of solutions to the coverage problems in wireless sensor networks, Journal of Internet Technology, 6(1), pp. 1-8.

[8] Liu J.J. (2011). Research on network coverage based on Voronoi graph in wireless sensor networks, Wuhan: Wuhan University of Technology.

[9] Li S.S. (2007). Research on key technologies for reliable data transmission in wireless sensor networks, Hunan: National University of defense technology.

[10] Kosanovic M., Stojcev M. (2008). Reliable transport of data in wireless sensor network, Proceedings of 26th International Conference on Microelectronics, pp. 455 - 458. https://doi.org/10.1109/ICMEL.2008.4559320

[11] Willig A., Karl H. (2005). Data transport reliability in wireless sensor networks - asurvey of issues and solutions, Praxis Der Informationsverarbeitung Und Kommunikation, 28, pp. 86 - 92. https://doi.org/10.1515/PIKO.2005.86

[12] Liudong X., Akhilesh S. (2006). Qos Reliability of Hierarchical Clustered Wireless Sensor Networks, Performance, Computing and Communications Conference 25th IEEE International, pp. 641-646.

[13] Zhou Z.H., Das S., Gupta H. (2004). Connected K-Coverage Problem in Sensor Networks Computer Communications and Networks, ICCCN, pp. 373-378.

[14] Ren Y., Zhang S.D., Zhang H.K. (2006). Coverage control theory and algorithm in wireless sensor networks, Journal of software engineering, pp. 422-433.

[15] Wang X., Xing G., Zhang Y. (2003). Integrated coverage andconnectivity configuration in wireless sensor networks, In: Akyildiz IF, Proc. of the ACM Int'l Conf. on Embedded Networked Sensor Systems (Sen Sys), pp. 28-39.

[16] Sen A., Shen B.H., Zhou L., Hao B. (2006). Fault-Tolerance in Sensor Networks: A New Evaluation Metric, Infocom IEEE International Conference on Computer Communications, 47, pp. 1-12. https://doi.org/10.1109/INFOCOM.2006.270

[17] Kashyap A., Khuller S., Shayman M. (2006). Relay Placement for Higher Order Connectivity in Wireless Sensor Networks, Infocom IEEE International Conference on Computer Communications, 15(1), pp. 1-12.

[18] Zhang S.J. (2010). Wireless sensor network technology and its application, Beijing: China Electric Power Press, pp. 210-213.

[19] Brunda S., Chaitra H.K. (2004). Survey of wireless sensor networks, Journal of communications, 25(4), pp. 114-124.

\section{$7 \quad$ Author}

Zhifu Luan is the lecturer of Weifang University of Science and Technology, Shandong, China. His research interest field is computer application technology. He has computer teaching experience of 20 years. He has presided over a scientific research project and participated in a few scientific research projects.

Article submitted 25 October 2017. Published as resubmitted by the author 28 November 2017. 